\title{
Ultrasound contrast agent loaded with nitric oxide as a theranostic microdevice
}

This article was published in the following Dove Press journal:

Drug Design, Development and Therapy

29 April 2015

Number of times this article has been viewed

\author{
Dmitry Grishenkov ${ }^{1-3}$ \\ Adrian Gonon ${ }^{3,4}$ \\ Eddie Weitzberg ${ }^{5}$ \\ Jon O Lundberg ${ }^{5}$ \\ Johan Harmark ${ }^{6}$ \\ Barbara Cerroni ${ }^{7}$ \\ Gaio Paradossi ${ }^{7}$ \\ Birgitta Janerot-Sjoberg ${ }^{1-3}$ \\ 'Department of Clinical Science, \\ Intervention, and Technology, \\ Karolinska Institutet, Stockholm, \\ Sweden; ${ }^{2}$ Department of Medical \\ Engineering, KTH, Royal Institute \\ of Technology, School of Technology \\ and Health, Stockholm, Sweden; \\ ${ }^{3}$ Department of Clinical Physiology, \\ Karolinska University Hospital, \\ Stockholm, Sweden; ${ }^{4}$ Department \\ of Medicine, Karolinska Institutet, \\ Stockholm, Sweden; ${ }^{5}$ Department \\ of Physiology and Pharmacology, \\ Karolinska Institutet, Stockholm, \\ Sweden; ${ }^{6}$ Department of Biosciences \\ and Nutrition, Karolinska Institutet, \\ KTH, Royal Institute of Technology, \\ School of Technology and Health, \\ Stockholm, Sweden; ' Department \\ of Chemical Sciences and Technologies, \\ University of Rome Tor Vergata, \\ Rome, Italy
}

Correspondence: Dmitry Grishenkov Karolinska Institutet, Department of Clinical Science, Intervention and Technology, Division of Medical Imaging and Technology, Alfred Nobels Alle 10, SE-I4I 52 Stockholm, Sweden

Tel +4687909720

Email dmitry.grishenkov@ki.se
Abstract: The current study describes novel multifunctional polymer-shelled microbubbles (MBs) loaded with nitric oxide (NO) for integrated therapeutic and diagnostic applications (ie, theranostics) of myocardial ischemia. We used gas-filled MBs with an average diameter of $4 \mu \mathrm{m}$ stabilized by a biocompatible shell of polyvinyl alcohol. In vitro acoustic tests showed sufficient enhancement of the backscattered power (20 dB) acquired from the MBs' suspension. The values of attenuation coefficient $(0.8 \mathrm{~dB} / \mathrm{cm} \mathrm{MHz})$ and phase velocities $(1,517 \mathrm{~m} / \mathrm{s})$ were comparable with those reported for the soft tissue. Moreover, polymer MBs demonstrate increased stability compared with clinically approved contrast agents with a fracture threshold of about $900 \mathrm{kPa}$. In vitro chemiluminescence measurements demonstrated that dry powder of NO-loaded MBs releases its gas content in about 2 hours following an exponential decay profile with an exponential time constant equal to 36 minutes. The application of high-power ultrasound pulse (mechanical index $=1.2$ ) on the MBs resuspended in saline decreases the exponential time constant from 55 to 4 minutes in air-saturated solution and from 17 to 10 minutes in degassed solution. Thus, ultrasound-triggered release of NO is achieved. Cytotoxicity tests indicate that phagocytosis of the MBs by macrophages starts within 6-8 hours. This is a suitable time for initial diagnostics, treatment, and monitoring of the therapeutic effect using a single injection of the proposed multifunctional MBs.

Keywords: microbubbles, contrast agent, ultrasound, nitric oxide, myocardial ischemia

\section{Introduction}

Continuous development of new therapeutic agents has made novel drugs available for a variety of diseases. However, a wide spectrum of active molecules alone does not guarantee optimal therapeutic efficacy, as other relevant factors, such as bioavailability and release mechanism, come into play. ${ }^{1}$ It can be proposed that the "drug" is not just a pharmacological substance anymore but rather a "complex" system whose enhanced efficacy is obtained by the synergic action of a chemical compound together with a controlled delivery system.

The current study introduces a new class of microdevices providing integrated therapeutic and diagnostic applications (ie, theranostics) of ischemic heart disease (IHD), the most widespread cardiovascular disease (CVD). ${ }^{2}$ IHD is the leading cause of death in both sexes, equally diagnosed in developed and developing countries, with mortality exponentially increasing with age. ${ }^{2}$ Efforts of a health care system should therefore be primarily focused on IHD prevention, timely detection, efficient differentiation, and instant treatment. Due to the aging population and recent medical interventions, survivors rapidly increase in number, and all measures taken to limit the myocardial damage will be beneficial to the patient, the health care system, and society.

Ultrasound is a most frequently used imaging modality for rapid bedside CVD diagnosis and monitoring. Intravenously injected ultrasound contrast agents (UCAs) 
significantly increase the diagnostic efficiency of myocardial ischemia and are currently used for perfusion and ventricular delineation/motion studies and more generally for passive enhancement of echoes produced by blood. ${ }^{3}$ UCAs are generally suspensions of gas-filled microbubbles (MBs) where the stabilizing shell of approved UCAs consists of either proteins $^{4}$ (eg, Optison ${ }^{\circledR}$ GE HealthCare) or phospholipids ${ }^{5}$ (eg, SonoVue ${ }^{\circledR}$, Bracco). In order to increase the circulation time and hence the effective imaging acquisition to several minutes, the gas core of these MBs consists of low soluble gas: sulphur hexafluoride $\left(\mathrm{SF}_{6}\right)$ in case of SonoVue ${ }^{\circledR}$ and perfluoropropane $\left(\mathrm{C}_{3} \mathrm{~F}_{8}\right)$ in an Optison ${ }^{\circledR}$ formulation. Nevertheless, even longer (ie, up to 30 minutes) circulation time would be desirable for molecular imaging using targeted or therapeutic MBs or for monitoring of the therapeutic effect. ${ }^{6}$ The conventional MBs thereby have limitations to act as drug carriers, and a relatively broad size distribution generates difficulties in optimizing ultrasound imaging ${ }^{7}$ and ultrasoundmediated drug delivery. ${ }^{8}$ Potentially, all the aforementioned limitations might be overcome by the application of novel polymer-shelled MBs. These MBs offer increased chemical and mechanical stability, can carry therapeutic payload, and can be fabricated with narrow size distribution for specific ultrasound imaging and controlled drug delivery.

Drugs for CVD that would profit from a local and controlled delivery overcoming systemic toxic effects are under development. ${ }^{9}$ Encapsulation of such drugs in biocompatible microcarriers (in our case MBs) is of particular interest. Following the ultrasound exposure, MBs will undergo controlled volumetric oscillations. Depending on the applied acoustic power, thermal and/or cavitation-related phenomena of various biological effects occur. For example, MBs in combination with ultrasound locally and transiently increase the permeability of biological barriers (ie, cell membranes). ${ }^{10}$ In the myocardium, acute reperfusion of an ischemic area results in increased cellular death of reversibly injured myocardial cells. Decreased bioavailability of nitric oxide (NO) and increased proinflammatory mediators are described as major actors also associated with patients with diabetes and atherosclerosis who are prone to develop ischemia. ${ }^{11}$ Cardioprotection during ischemia and reperfusion is essentially dependent on intact $\mathrm{NO}$ production and release. ${ }^{12} \mathrm{NO}$ mediates vasorelaxation ${ }^{13}$ and inhibits the clotting of thrombocytes $^{14}$ as well as the accumulation of white blood cells in the injured tissue. ${ }^{15}$ Systemic treatment with NO or an NO donor, however, has a narrow therapeutic window and may provoke, for example, critical blood pressure drop, albeit the half-life of NO in blood is short due to rapid oxidation by oxyhemoglobin. ${ }^{16}$ As a result, local, specific, and controlled delivery of NO is of great clinical interest but currently unrealizable with commercially available drug carriers. It is worth mentioning that exposure to low-intensity ultrasound alone reduces ischemia reperfusion injury through reduced vasoconstriction, leukocyte adhesion, oxidative stress, and increased capillary perfusion and NO level due to enhanced shear stress. ${ }^{17}$ These effects can be further extended with the presence of MBs. ${ }^{18}$ Here, we describe a theranostic approach for management of myocardial reperfusion injury, utilizing ultrasound to enhance NO release from polymer MBs enriched with NO for local vascular delivery.

The pioneering work by Postema et $\mathrm{al}^{19}$ discusses through mathematical modeling the potential limitations of NO delivery based on the retrospective analysis of other types of nitrogen $\left(\mathrm{N}_{2}\right)$ - and air-filled contrast agents: PB127 (POINT Biomedical Corp.) and Quantison (Upperton Ltd). It was suggested that successful NO delivery can only be achieved by ultrasound cracking of the MBs in red blood cell-free plasma close to endothelium. The main goal of the current study is to further develop the novel multifunctional MB platform through structural, acoustic, and toxicity evaluation, in order to demonstrate the strategy of therapeutic gas loading and experimentally assess the release profile, focusing on NO and IHD. It is a first step toward development of a multifunctional theranostic device for combined diagnostics and ultrasoundmediated delivery of a therapeutic gas for IHD.

\section{Materials and methods}

Gas-filled MBs stabilized by a biocompatible polyvinyl alcohol (PVA) shell are fabricated according to the previously developed protocol. ${ }^{20}$ In brief, the PVA solution $(2 \% \mathrm{w} / \mathrm{v})$ is oxidized with sodium metaperiodate to obtain short telechelic PVA chains. The crosslinked stabilizing PVA shell of the MBs is formed at the water/air interface under strong shear stress using an UltraTurrax homogenizer (IKA Werke GmbH, Staufen, Germany) equipped with a $25 \mathrm{G}$ Teflon tip operating at 8,000 rpm for 2 hours. This reaction is carried out at $\mathrm{pH} 5$ at room temperature.

The resulting MBs have a gas core that allows them to efficiently scatter ultrasound waves. By substituting the core gas with a therapeutic gas, in our case NO, new possibilities for local, specific drug delivery triggered by ultrasound are offered.

\section{Therapeutic gas loading}

After fabrication of the suspension of plain air-filled MBs (protocol described previously), ${ }^{20}$ freeze-drying of the 
suspension was performed using rapid cooling with liquid nitrogen and a vacuum pump for efficient ice evaporation. The resulting PVA powder, consisting of oval capsules, was enclosed into a pressure chamber where air was first exchanged with $\mathrm{N}_{2}$ gas (AGA AB, Lidingö, Sweden) for 5 minutes at 1 bar overpressure. $\mathrm{N}_{2}$ was considered to be unreactive at the levels of pressures and temperatures employed in this study. It was introduced in the chamber before NO loading in order to avoid production of nitrogen dioxide due to rapid oxidation reaction between $\mathrm{NO}$ and air. Thereafter, $\mathrm{NO}$ (AGA AB, Lidingö, Sweden) was introduced in the pressure chamber at 2 bars during 2 hours for the capsule loading. The final step was to flush the pressure chamber with $\mathrm{N}_{2}$ in order to remove excess NO. Finally, the obtained dry MB powder was studied as such or after resuspension in water. It can be noted that the total amount of NO per milliliter of suspension is strongly dependent not only on the loading efficiency but also on the $\mathrm{MB}$ concentration in the resuspended media.

$\mathrm{NO}$ and $\mathrm{N}_{2}$ were purchased from AGA AB (Lidingö, Sweden).

\section{Structural characterization}

A confocal laser scanning microscope, Eclipse Ti-E (Nikon Instruments Europe B.V., Amsterdam, the Netherlands), equipped with Argon ion laser operated at $488 \mathrm{~nm}$ (Melles Griot Rochester, NY, USA), He-Ne source operated at 543.5/633 nm (Spectra-Physics, Santa Clara, CA, USA), and a $60 \mathrm{x} / 1.4$ oil immersion objective were used to visualize and assess the structural properties of the MBs. Tests carried out with fluorescent giant liposomes explored a resolution of $0.5 \mu \mathrm{m}$ for the instrument. The tetramethylrhodamine isothiocyanate (TRITC) was used for fluorescent labeling of the MBs. External diameter and shell thickness were determined by focusing on the MBs' equatorial plane.

A scanning electron microscope, XL30 ESEM (Philips, Eindhoven, the Netherlands), equipped with tungsten filament as an electron source and both gaseous secondary electron and backscattered electron detectors were employed to produce images in low and high vacuum mode. Tests were carried out on a dry powder of MBs at a high vacuum mode and on a wet sample of either original or resuspended MBs at a low vacuum or environmental (ESEM) mode.

A transmission electron microscope (TEM) JEM2100F (JEOL, Tokyo, Japan), operating at an accelerating voltage of $200 \mathrm{kV}$, was employed to assess the structural morphology of PVA MBs. Sample preparation is similar to that described previously in Brismar et $\mathrm{al}^{21}$ and includes MB trapping in a 10\% gelatin solution (Merck, Darmstadt,
Germany), embedding the obtained pellet in epoxy resin LX 112 (Ladd, Burlington, VT, USA), sectioning into $50 \mathrm{~nm}$ slices with Leica Ultracut UCT (Leica, Vienna, Austria), and finally MB placement on carbon-coated copper grids for imaging. Micrographs were recorded on a Tietz (TVIPS $\mathrm{GmbH}$, Gauting, Germany) charge-coupled device (CCD) camera.

By applying a TEM, CM120 (Philips), operating at an accelerating voltage of $120 \mathrm{kV}$, the structural integrity of the MBs was assessed before and after ultrasound exposure using an acoustic wave with an amplitude well above the MB-fracturing pressure threshold. Sample preparation includes negative staining with $1 \%$ uranyl acetate applied to a carbon-coated glow-discharged copper grid. Micrographs were recorded on a $1 \mathrm{k}$ Tietz (TVIPS GmbH) CCD camera.

\section{Biotoxicity evaluation}

In vitro cytotoxicity of the PVA shelled MBs was tested on a mouse leukemic monocyte macrophage cell line (RAW 264.7) that was obtained from Instituto Sperimentale Zoprofiattico della Lombardia e dell'Emilia Romagna (Italy). The cells were kept in Dulbecco's Modified Eagle's Medium supplemented with $2 \mathrm{mM}$ L-glutamine, $10 \%$ heat-inactivated fetal bovine serum, and $1 \%$ penicillin/streptomycin solution at $37^{\circ} \mathrm{C}$ in a $5 \% \mathrm{CO}_{2}$ atmosphere. Internalization or uptake of the MBs by the macrophages was evaluated using two experimental scenarios. The first scenario included labeling of the MBs with fluorescein isothiocyanate (FITC) while RAW 264.7 cells were left unlabeled. After incubation of cells with MBs for 2 hours, the sample was treated with Trypan Blue $(0.1 \mathrm{mg} / \mathrm{mL})$ solution and visualized using both transmitted light and confocal laser scanning microscopy (CLSM). The second scenario includes labeling of the MBs with TRITC while RAW 264.7 cells were stained with FITC-phalloidin to visualize the actin cytoskeleton of the microphages. After incubation of cells with MBs for 8 hours, the sample was visualized using CLSM.

\section{Acoustic characterization}

A nondestructive evaluation was performed in order to assess the acoustic response from the MBs' suspension. This task was carried out according to the previously developed protocol $^{22}$ that utilizes a broadband pulser-receiver, PR5072 (Panametrics, Waltham, MA, USA), operating at pulse repetition frequency of $1 \mathrm{kHz}$, and a digital oscilloscope, TDS 5052 (Tektronix Inc, Beaventor, OR, USA). To cover the typical frequency range of clinical ultrasound (1-15 MHz), 
three probes with nominal frequencies of $2.25,5$, and $10 \mathrm{MHz}$ were employed. The bandwidths corresponding to -6 and $-20 \mathrm{~dB}$ acoustic output are presented in Table 1. These transducers produced a pressure field not larger than $100 \mathrm{kPa}$ at a distance corresponding to the near-field length.

At low excitation pressure, below $100 \mathrm{kPa}$, the backscattered power, attenuation coefficient, and phase velocity were determined at specified MB concentration. Based on the results from backscattered power tests carried out using a $5 \mathrm{MHz}$ transducer, dose-response curves were constructed. Attenuation coefficient and phase velocity tests aim to determine the resonance frequency of the MBs' oscillation, and thus all three transducers covering the frequency range from 0.7 to $14.5 \mathrm{MHz}$ were employed. The spectrum of the time domain signals was averaged over ten samples to reduce the noise level by approximately $10 \mathrm{~dB}$. Each data point represents the average value over three independent measurements, and the error bars show standard deviation.

A suspension of the MBs diluted to the concentration that leads to maximum backscattered power and the values of attenuation coefficient and phase velocity comparable with the values reported for the soft tissue is used in the next step. Here, fracturing of the MBs' shell at high excitation pressure, typically above $500 \mathrm{kPa}$, was evaluated using the cross-correlation method described previously by Pecorari and Grishenkov. ${ }^{23}$

Briefly, two transducers with the central frequencies 2.5 and $5 \mathrm{MHz}$ were used as an emitter and receiver, respectively. The bandwidths corresponding to -6 and $-20 \mathrm{~dB}$ acoustic output are presented in Table 1. The emitting transducer was excited by a system for the Study of Nonlinear Acoustic Phenomena (SNAP Mark IV; Ritec Inc., Warwick, RI, USA) that allows control of the excitation intensity, frequency, number of cycles, and pulse repetition frequency of each ultrasound pulse. For the evaluation of the fracturing pressure threshold, the emitting transducer is excited by a ten-cycle sinusoidal burst at varied electrical intensity. The peak negative pressure in the focal region of the emitting transducer assessed by $0.075 \mathrm{~mm}$ needle hydrophone
(Precision Acoustic, Dorchester, UK) was increased stepwise from 0.2 to $2.5 \mathrm{MPa}$. For each value of the peak negative pressure, 100 time-domain signals are acquired. Next, for each pair of consecutive signals, the maximum value of the correlation coefficient $\max |\rho(\tau \mid T)|$ is computed. Then, the average correlation coefficient $\langle\max |\rho(\tau \mid T)|\rangle$ is calculated for this particular pressure level.

The value of $\langle\max |\rho(\tau \mid T)|\rangle$ equal to 0.95 was assigned as a fracturing threshold, from which the peak negative pressure was identified.

\section{Assessment of gas content}

Assessment of the gas content and gas dissolution rate from the dry NO-loaded MBs was performed using an ozone-based chemiluminescence analyzer (CLD 77 am NO analyzer, Eco Physics AG, Duernten, Switzerland) operating at a $300 \mathrm{~mL} / \mathrm{min}$ flow rate. A syringe filter with a pore size of $0.2 \mu \mathrm{m}$ was employed to eliminate the involuntary liquid flow into the analyzer. Every 5 minutes, a $10 \mathrm{~mL}$ gas sample was extracted with a syringe from the otherwise sealed $50 \mathrm{~mL}$ Falcon tube and was subjected to analysis. Continuous recordings digitized at a rate of 100 sample/min were performed to monitor the instantaneous NO release to the atmosphere above the dry sample. Areas under the curve from number of counts versus time were calculated and reveal the total NO content at any specific time point. Since the total amount of MBs in each vial was difficult to estimate for further comparative analysis, acquired data were normalized to the maximum value in a release profile, typically achieved at the first measurement time point. The procedure was repeated every 5 minutes during 2 hours.

The same protocol was employed in an experiment with NO-loaded MBs resuspended in saline. Degassed as well as standard (ie, air saturated) saline solutions were used. In total, four vials with the suspension of the NO-loaded MBs were prepared. Two vials were used as a control to assess the passive release of NO to the degassed and air saturated solution, respectively. Another two vials were used to estimate the effect of high-intensity ultrasound burst on the NO release

Table I Characteristics of the ultrasound transducers

\begin{tabular}{llllll}
\hline $\begin{array}{l}\text { Center frequency, } \\
\mathbf{M H z}\end{array}$ & $\begin{array}{l}-\mathbf{6 ~ d B} \text { bandwidth, } \\
\mathbf{M H z}\end{array}$ & $\begin{array}{l}-\mathbf{2 0 ~ d B} \text { bandwidth, } \\
\mathbf{M H z}\end{array}$ & $\begin{array}{l}\text { Aperture diameter, } \\
\mathbf{m m}\end{array}$ & $\begin{array}{l}\text { Panametrics } \\
\text { part no }\end{array}$ \\
\hline 2.25 & $1.4-2.8$ & $0.7-3.5$ & 13 & V-306-SU & Flat \\
5.0 & $3.1-6.7$ & $1.2-8.1$ & 13 & V-309-SU & Flat \\
10.0 & $6.3-12.1$ & $2.5-14.5$ & 13 & V-3II-SU & Flat \\
2.5 & $1.8-3.4$ & $1.5-4.6$ & 13 & Krautkramer, Gamma Series & Focused \\
5.0 & $3.4-6.8$ & $1.9-8.5$ & 13 & V-309-SU & Focused \\
\hline
\end{tabular}


profile in degassed and air-saturated saline solution. A clinical ultrasound scanner, Vivid I ${ }^{\circledR}$ (GE Healthcare, Wauwatosa, WI, USA), equipped with a linear transducer (8L-RS, GE Parallel Design Inc., Phoenix, AZ, USA) operating in contrast octave mode $(4.4 / 7.9 \mathrm{MHz})$ was employed. High-power ultrasound bursts (mechanical index $[\mathrm{MI}]=1.2$ and soft-tissue thermal index $=0.9$ ) following at a rate of 32 frames per second were used in order to ensure the fracture of the MBs not only at a focus length of $1.75 \mathrm{~cm}$ but also through the whole diameter of the $50 \mathrm{~mL}$ Falcon tube. To minimize the reflection and scattering from the cylindrical plastic wall of the tube, a small acoustic window was cut and sealed with the latex film.

The presence of nitrate and nitrite as oxidation products following the release of NO in standard and degassed saline was analyzed using high-performance liquid chromatography (ENO-20 Eicom, Kyoto, Japan) as previously described in detail. ${ }^{24}$ In brief, the system uses reverse-phase chromatography to separate nitrite from nitrate. Nitrate was then reduced to nitrite in a reaction with cadmium and reduced copper inside a reduction column. Reduced nitrite was then derivatized with Griess reagent, and a detector at $540 \mathrm{~nm}$ was used to measure the level of diazo compounds. Liquid samples $(100 \mu \mathrm{L})$ were extracted before the application of ultrasound, after 30 minutes and 60 minutes of ultrasound exposure.

\section{Results and discussion Structural properties}

In general, the dimension of MBs composing UCAs is very important, as they should be smaller than the capillary lumen $^{25}$ (ie, about $7 \mu \mathrm{m}$ ) in order not to obstruct the blood flow. In addition, MBs should be larger than hundreds of nanometers in order to avoid the response of the immune system. ${ }^{26}$ An ideal UCA is a monodisperse suspension of the gas-filled MBs that are not only acoustically echogenic but also mechanically and chemically stable. CLSM tests that assess equatorial planes of about $150 \mathrm{MBs}$ reveal that these novel MBs fabricated at $\mathrm{pH} 5$ and room temperature have an external diameter of $3.8 \pm 0.8 \mu \mathrm{m}$ and shell thickness approximately $0.4 \pm 0.1 \mu \mathrm{m}$.

Previous studies have demonstrated that polymer-shelled MBs can be produced with different average sizes and shell thicknesses by altering the $\mathrm{pH}$ and temperature of the master solution. For example, the external diameter increases from $2.0 \pm 0.3$ to $4.4 \pm 1.1 \mu \mathrm{m}$ when the master solution temperature increase from $4^{\circ} \mathrm{C}$ to $37^{\circ} \mathrm{C}$ and shell thickness increases from $0.4 \pm 0.1$ to $0.6 \pm 0.1 \mu \mathrm{m}$ when $\mathrm{pH}$ decreases from 5 to $2 .{ }^{27}$ Application of a homogenizer's tip with a fine gap (25F instead of $25 \mathrm{G})$ decreases both the external diameter and its polydispersity from $3.2 \pm 0.9 \mu \mathrm{m}$ when using a $25 \mathrm{G}$ tip to $2.0 \pm 0.4 \mu \mathrm{m}$ for the $25 \mathrm{~F}$ tip. ${ }^{28} \mathrm{MBs}$ with narrow size distribution (ie, monodisperse suspension) have a unique resonance frequency of oscillation. Acoustic insonation at a frequency close to the resonance generates a strong nonlinear harmonic response, which can be easily discriminated in the received echo. ${ }^{29}$

In Figure 1A, the TEM image depicts a typical MB geometry, and shell constitution is present. Pixel gray values presented in Figure 1B were acquired along the yellow line in Figure 1A. The pixel gray value increases sharply at about $400 \mathrm{~nm}$, indicating the inner border of the shell. From 400 to $800 \mathrm{~nm}$, the pixel gray value remains nearly constant, representing the hard or compacted part of the shell. Worth noting is that the pixel gray value decreases gradually from 800 to $1,000 \mathrm{~nm}$, indicating the soft or noncompacted part of the shell. Cavalieri et $\mathrm{al}^{30}$ observed a similar shell composition and refer to the soft part of the shell as a "hairy" structure that constitutes primarily microfibrils and air- and/ or liquid-filled pockets.
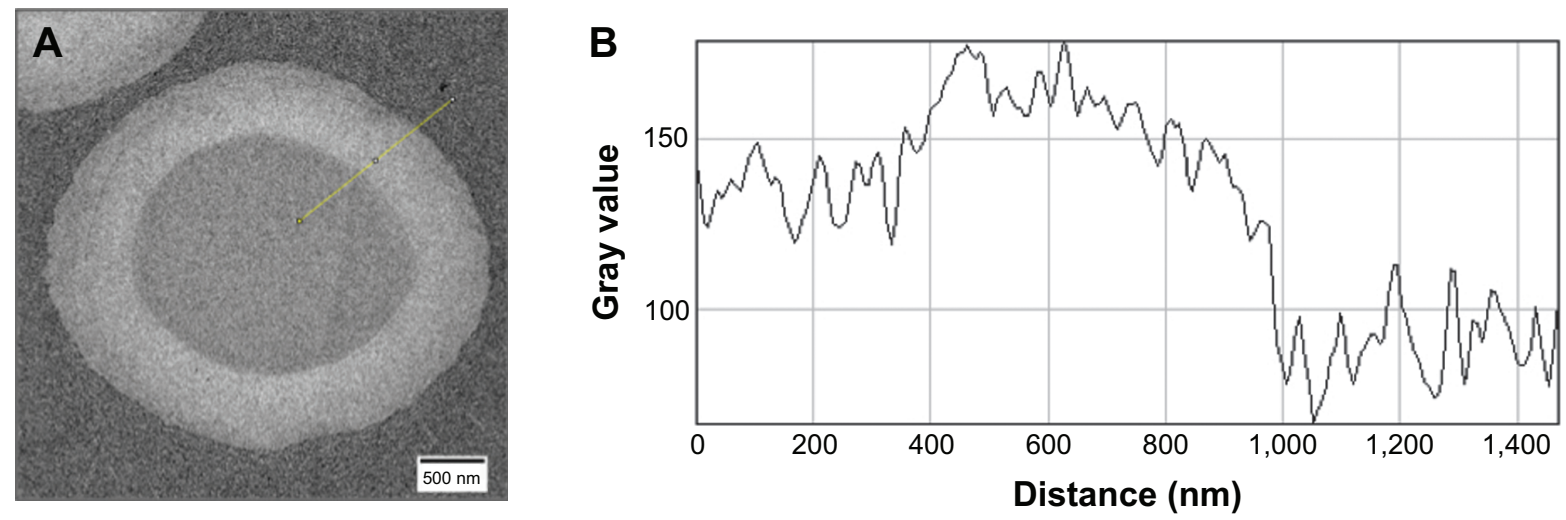

Figure I Transmission electron micrograph of plain gas-filled microbubble (A). Pixel gray values $(\mathbf{B})$ are measured along the yellow line. Note that the hard shell is from 400 to $800 \mathrm{~nm}$, while the soft shell is from 800 to $1,000 \mathrm{~nm}$. 
A

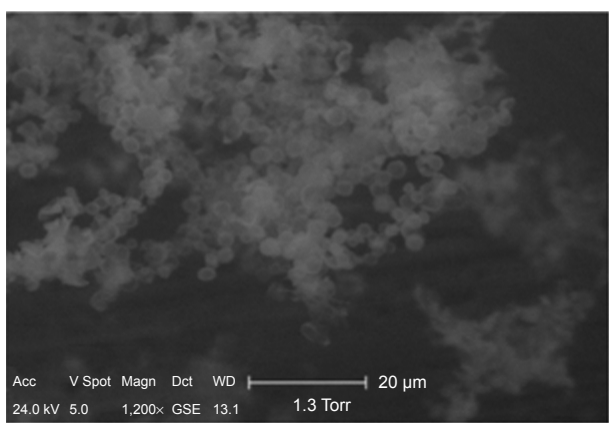

B

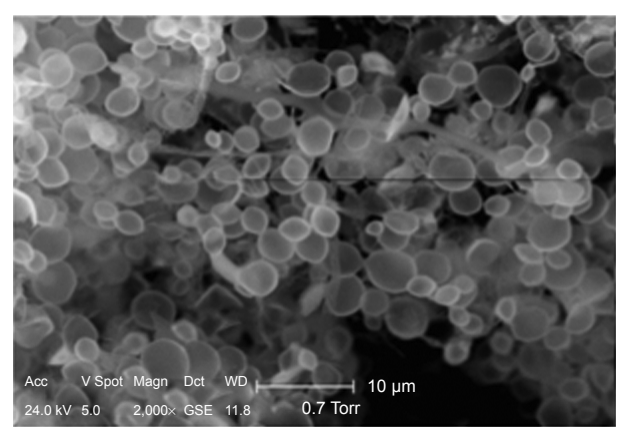

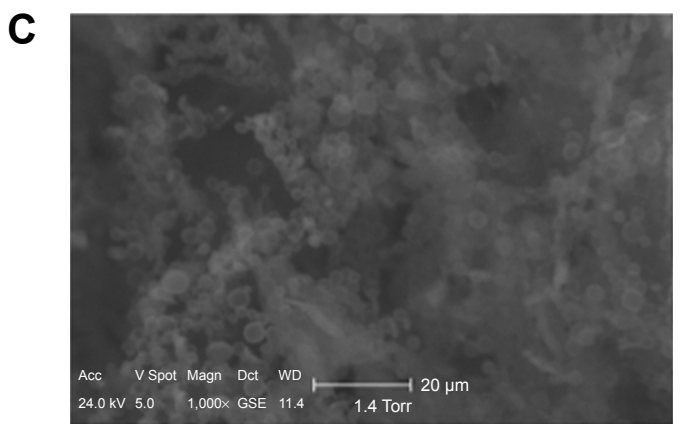

Figure 2 Scanning electron micrograph of the microbubble suspension before (A), immediately after freeze-drying (B), and after redispersion in distilled water (C).

The ability to reconstitute MBs in a buffer solution after freeze-drying is demonstrated in Figure 2A-C. It can be noted that MBs retain their size and spherical shape most probably due to the $400 \mathrm{~nm}$ thick crosslinked polymer shell. ${ }^{31}$ In addition, preliminary tests indicate that the acoustic response from the suspension exposed to the freeze-drying/ redispersion process is similar to that acquired from the control intact suspension.

\section{Biotoxicity properties}

In Figure 3, an example of fluorescently labeled MBs being phagocytosed by a macrophage (RAW 264.7) is shown.

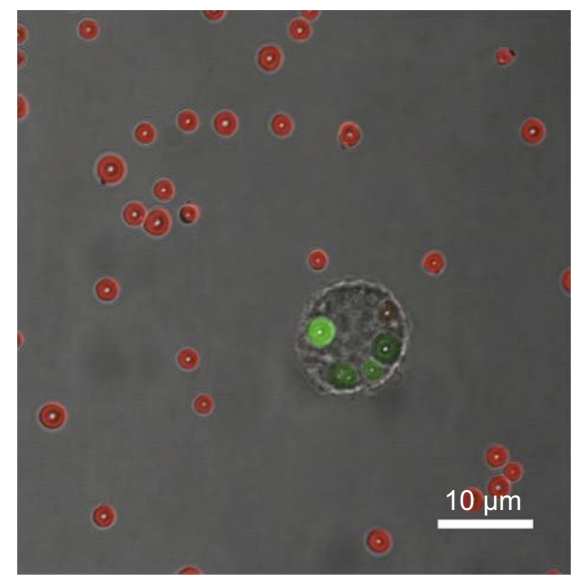

Figure 3 Merged light microscopy and confocal laser scanning microscopy images of RAW 264.7 incubated with microbubbles for 2 hours.
Following the first experimental scenario, the sample is treated with Trypan Blue solution, and only the internalized MBs expose green color. Trypan Blue is able to quench the green fluorescence of the FITC molecules conjugated to the PVA MBs. Due to the inability of the dye to penetrate the cell membrane, ${ }^{32}$ the green fluorescent spots are attributed to particles internalized in the cytoplasm of the cells. The red fluorescent spots surrounding the cells indicate the interaction of Trypan Blue with bovine serum albumin (BSA) molecules contained in the culture medium and deposited as a protein corona around the PVA MBs. As reported in literature, the dye-BSA complex induces a fluorescence in the emission spectrum of Trypan Blue at $670 \mathrm{~nm} .{ }^{32}$

Following the second experimental scenario, macrophages (RAW 264.7) were incubated with MBs for 6 hours. Figure 4 provides the evidence that phagocytosis events are completed already after 6 hours of incubation. CLSM allows detection of fluorescently labeled TRITC (red) MBs inside the cell in which actin cytoskeleton was labeled with FITC-phalloidin and appears green in the figure. Both single bubbles and clusters of MBs can be observed in the cytoplasm of the cells.

A previous in vitro study ${ }^{33}$ of macrophage response showed that internalization of MBs occurs in a period between 4 and 8 hours, and that macrophage viability, assessed using live/dead assay, is not influenced by the presence of MBs within the time of the experiment. 


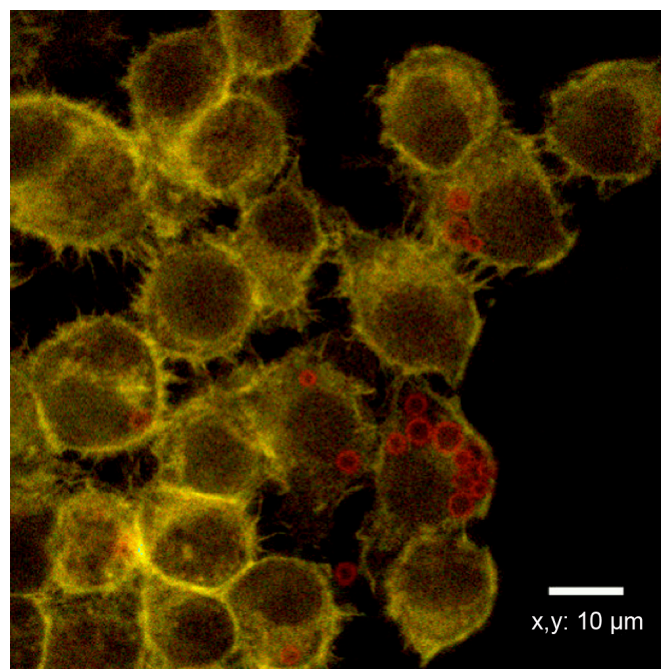

Figure 4 Incubation of 6 hours between RAW 264.7 and microbubbles. Cells are stained with fluorescein isothiocyanate phalloidin (green) while microbubbles are tetramethylrhodamine isothiocyanate (red) labeled. Cellular uptake is observed.

To summarize, the cell viability tests performed so far indicate that PVA MBs are biocompatible and that there is apparent immune system response after $6-8$ hours. This is a suitable time for initial diagnostics, ultrasound-mediated drug delivery, and monitoring of the therapeutic effect of the treatment followed by a single injection of the multifunctional contrast agent.

Recent experiments carried out by intravenous administration of PVA MBs in mice have shown complete biocompatibility with absence of toxicity sign as weight loss or influence on motility. In order to investigate the distribution of the MBs in different organs, a histological study in combination with MRI pharmacokinetic modeling was performed. Most of the MBs were found in the capillaries or phagocytosed by macrophages, preferably in the liver and spleen. ${ }^{34}$

A

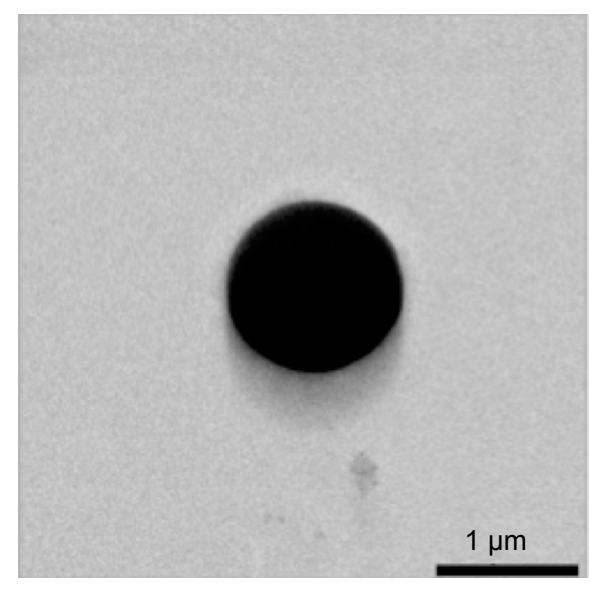

To address our MB biodegradability issue, TEM was performed. Figure 5A and B depicts a typical example of TEM of a single suspended $\mathrm{MB}$ of about $1 \mu \mathrm{m}$ before and after exposure to a high-power ultrasound burst of $\mathrm{MI}=1.6$. Worth noting is that after the ultrasound burst, the MB shell remains almost intact, with multiple cracks formed in the big shell fragment.

\section{Acoustic properties}

Figure $6 \mathrm{~A}-\mathrm{C}$ reports the results of in vitro acoustic tests, backscattered power, attenuation coefficient, and phase velocity, performed on MB suspensions of varied concentrations, from $5 \times 10^{5}$ to $10^{7} \mathrm{MB} / \mathrm{mL}$.

An increase of backscattered power (Figure 6A) from about 10 to $20 \mathrm{~dB}$, followed by an enhancement plateau, was observed with increasing MB concentration. A similar dose-response profile with a plateau value of enhancement of about $20 \mathrm{~dB}$ was reported in a previous study on other types of polymer-shelled MBs. ${ }^{35,36}$

A monotonic increase of attenuation coefficient (Figure 6B) and phase velocity (Figure 6C) within the investigated frequency range from 0.7 to $14.5 \mathrm{MHz}$ was observed for all concentrations considered in this study. The measurements performed at $10 \mathrm{MHz}$ demonstrate the increase of the attenuation coefficient from $1 \mathrm{~dB} / \mathrm{cm}$ to $30 \mathrm{~dB} / \mathrm{cm}$ and the decrease of phase velocity from $1,523 \mathrm{~m} / \mathrm{s}$ to $1,505 \mathrm{~m} / \mathrm{s}$ when the $\mathrm{MB}$ concentration increased from $8 \times 10^{4}$ to $1 \times 10^{7} \mathrm{MB} / \mathrm{mL}$. This finding is in agreement with results reported in our previous study ${ }^{33}$ and the general theory of the scattering of acoustic waves, ${ }^{37}$ where an increasing amount of scatterers leads to an increase of the total power being both absorbed and scattered by MB suspension. Further visual inspection does not reveal any apparent sign of the sharp peak in the attenuation spectra

B

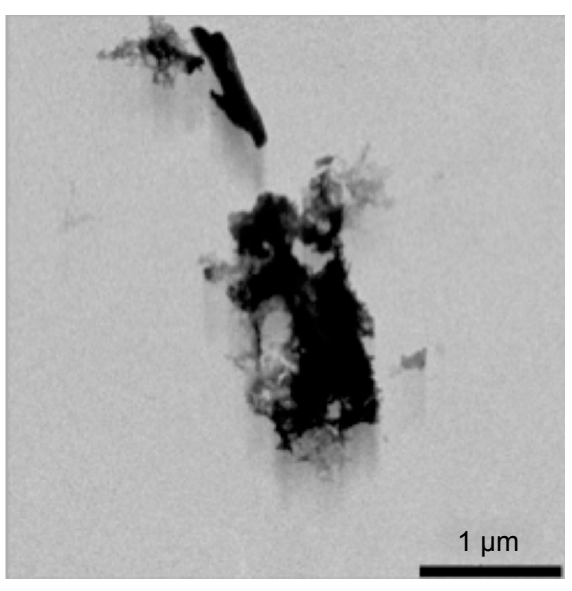

Figure 5 Transmission electron microscope micrograph of polyvinyl alcohol microbubble before (A) and after (B) high-power ultrasound exposure. 
A

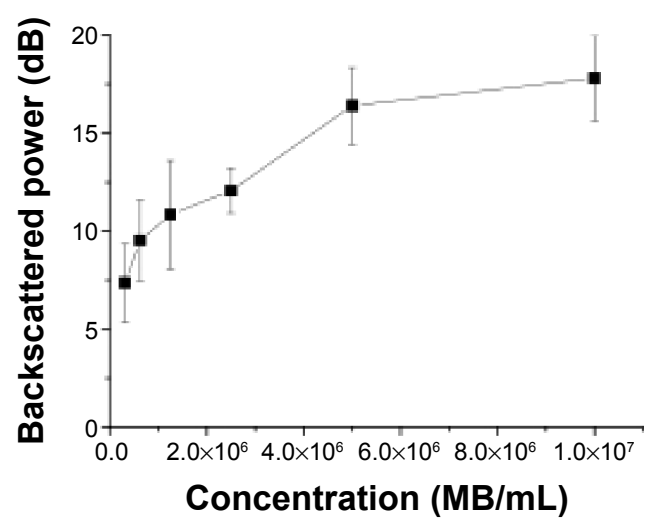

B

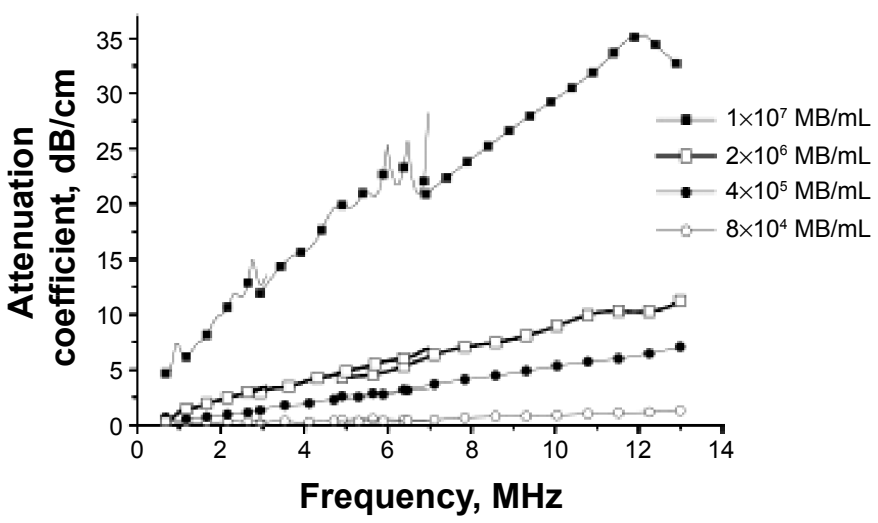

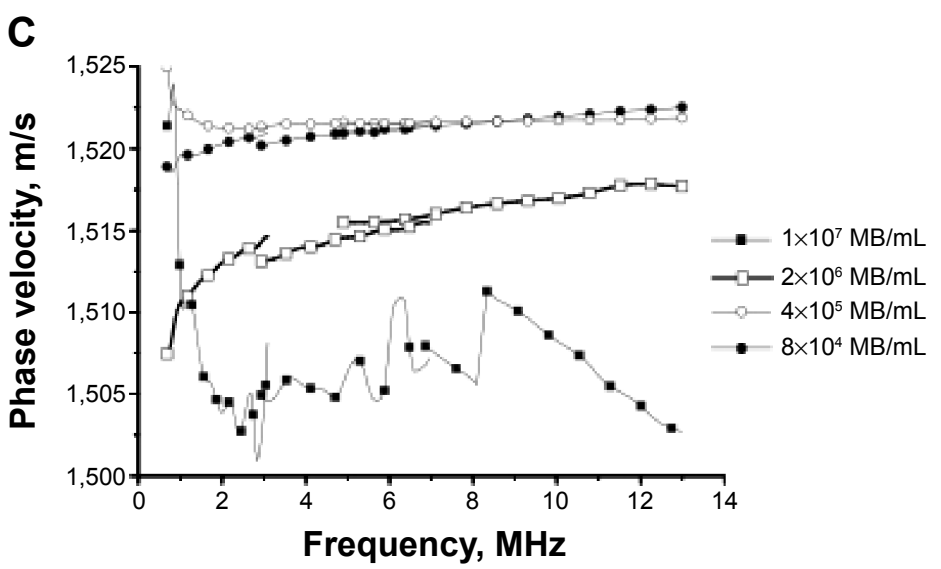

Figure 6 In vitro acoustic characterization: (A) backscattered power, (B) attenuation coefficient spectra, and (C) phase velocity spectra at various microbubble (MB) concentrations.

(Figure 6B) or discontinuities in the phase velocity spectra (Figure 6C), which are typically associated with the resonating MBs. This allows us to hypothesize that the resonance frequency of the oscillating MB either is higher than $14.5 \mathrm{MHz}^{38}$ or the resonance peak is heavily damped and not possible to visually identify ${ }^{39}$ Both suggestions are most probably related to the MBs' geometry where small MBs with a mean diameter of $3.8 \mu \mathrm{m}$ are enclosed in a relatively thick polymer shell of $400 \mathrm{~nm}$. A similar finding (ie, increase of the resonance frequency and broadening of the spectra) was reported by Hoff ${ }^{40}$ when evaluating thin-shelled polymer MBs with size of about $3.5 \mu \mathrm{m}$.

Worth noting is that a concentration of $2 \times 10^{6} \mathrm{MB} / \mathrm{mL}$ values of attenuation coefficient of about $8 \mathrm{~dB} / \mathrm{cm}$ and phase velocity of about $1,517 \mathrm{~m} / \mathrm{s}$ obtained at $10 \mathrm{MHz}$ and $37^{\circ} \mathrm{C}$ are comparable with those typically reported for the soft tissue $^{41}$ (ie, 5-10 dB/cm and 1,500-1,600 m/s, respectively). As a result, this concentration was further employed in the next study examining the level of peak negative pressure, $\mathrm{P}_{\text {thr }}$, at which the polymer shell starts to fracture. All tests performed with a varied number of cycles in the pulse and pulse repetition frequency report similar findings that fatigue, accumulation of the damage within the shell, is the major mechanism responsible for the shell fracturing. A $\mathrm{P}_{\text {thr }}$ below $900 \mathrm{kPa}$ at $2.25 \mathrm{MHz}(\mathrm{MI}=0.6)$ never fractures the shell. In comparison, Marmottant et al ${ }^{42}$ report on the commercially available phospholipid-shelled contrast agent SonoVue ${ }^{\circledR}$ (Bracco) fracturing onset at $300 \mathrm{kPa}$ at $2 \mathrm{MHz}(\mathrm{MI}=0.2)$ and further emphasize the increased stability of this novel MB.

To summarize, the proposed polymer-shelled MBs offer increased mechanical stability with prolonged blood circulation time - properties favorable for carrying highly soluble gas such as air, oxygen, and NO. In general, the knowledge of $\mathrm{P}_{\text {thr }}$ is of great importance if a destruction mechanism will be considered for the release of therapeutic substances. Moreover, the destruction/replenishment algorithm, ${ }^{43}$ typically performed at a pressure value above $\mathrm{P}_{\text {thri }}$, offers additional possibilities to study therapeutic effects on tissue perfusion using intact MBs recirculating to the region of interest.

\section{Therapeutic gas release}

Release of NO gas from the loaded PVA MBs was first assessed directly from the dry powder using a 
A

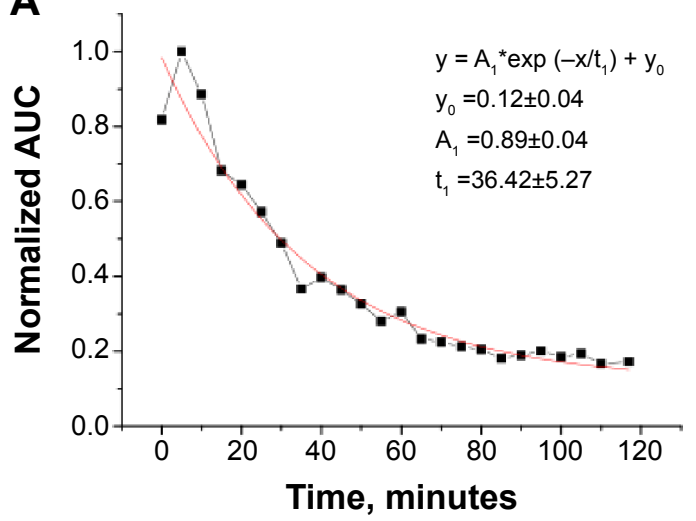

B

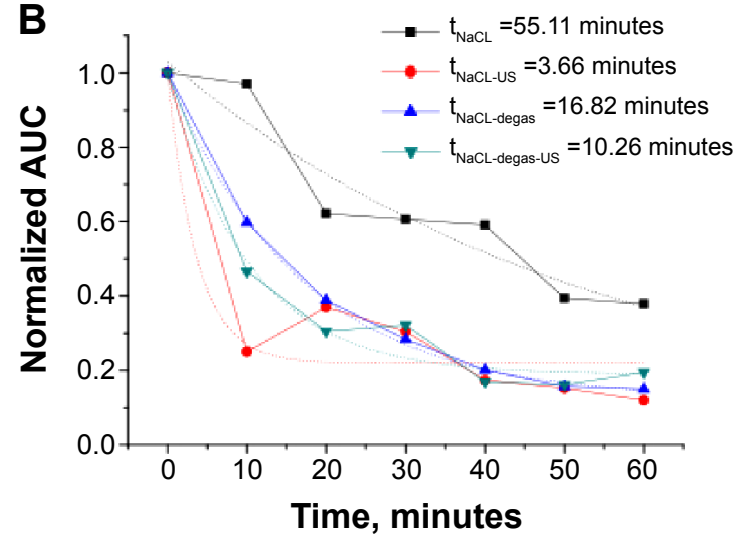

Figure 7 Release profile of NO from the dry powder (A) and redispersed in physiological solution (B) microbubbles. Ultrasound-mediated release of NO is observed for both standard $(\mathrm{NaCl})$ and degassed $(\mathrm{NaCl}$-degas) physiological solutions.

Notes: Exponential function of a time $y=A_{1}{ }^{*} \exp \left(-x / t_{1}\right)+y_{0}$ were used to quantify the gas release. $A_{1}$ represents the initial NO concentration at $t=0$; $t_{1}$ represents mean half-time of the gas release; $y_{0}$ is a plateau value that represents the remaining $N O$ concentration at $t=\infty$.

Abbreviations: NO, nitric oxide; AUC, area under the curve.

chemiluminescence method. In Figure 7A, the typical exponential decay profile of the release curve is shown, with an exponential time constant that represents mean half-time, equal to 36 minutes. The release curve flattened out at about 80 minutes, reaching a plateau at about 120 minutes. A similar release profile demonstrated in Figure 7B was obtained when dry powder was resuspended in saline $(0.9 \% \mathrm{NaCl})$ and the NO release monitored in the atmosphere immediately above the liquid surface. The effect from the application of the ultrasound pulse with $\mathrm{MI}=1.2$ that is supposed to fracture the MBs was investigated for the powder resuspended in standard (labeled as $\mathrm{NaCl}$ ) and degassed (labeled as $\mathrm{NaCl}-$ degas) saline solutions. Worth noting is that application of ultrasound pulse decreases the exponential time constant from 55 to 4 minutes in standard saline and from 17 to 10 minutes in degassed saline, respectively. It indicates a possibility for triggered ultrasound-mediated release of NO from the MB suspension. Such an effect is more pronounced when a standard physiological solution was used (reduction of the time constant from 55 to 4 minutes, Figure 7B), making further clinical studies in whole blood even more encouraging. In case of degassed saline, the effect of ultrasound is somehow blurred, probably due to passive diffusion of $\mathrm{NO}$ to the solution but not to the atmosphere above, in which measurements are made.
Results from the nitrite and nitrate measurements in the MB suspension before and after 30 and 60 minutes of ultrasound exposure are presented in Table 2. Values reported at time zero indicate the instantaneous release of NO to the solution and are followed by a moderate increase in both nitrite and nitrate production due to ultrasound exposure. Higher values reported in degassed saline indicate that $\mathrm{NO}$ is leaving MBs into the solution in a faster rate. This is in qualitative agreement with the finding reported from the gas phase assessment where a smaller (55 vs 16 minutes) exponential time constant was observed in case of degassed saline.

One of the potential clinical applications of NO-loaded MBs (ie, possibility to prevent clot formation) was described in a previous in vitro study by Cavalieri et al. ${ }^{30}$ It was demonstrated that after 1 hour following the NO loading, MBs still retain some $\mathrm{NO}$ and a clot is not formed. However, most NO was released from the powder within 2 hours and a clot started to develop.

\section{Conclusion}

The translational significance of this study is to develop a simple combined tool for diagnostics and treatment of acute myocardial ischemia in patients with atherosclerosis or diabetes with impaired bioavailability of NO. Keeping in mind that systemic treatment with an NO donor has a very narrow therapeutic window and may provoke critically low blood

Table 2 Production of nitrite and nitrate in the suspension

\begin{tabular}{|c|c|c|c|c|}
\hline \multirow[t]{2}{*}{ Time, mins } & \multicolumn{2}{|l|}{$\mathrm{NaCl}$} & \multicolumn{2}{|l|}{$\mathrm{NaCl}$-degas } \\
\hline & Nitrite, $\mu \mathrm{M}$ & Nitrate, $\mu \mathrm{M}$ & Nitrite, $\mu \mathbf{M}$ & Nitrate, $\mu \mathrm{M}$ \\
\hline 0 & 0.363 & 74.630 & 0.296 & 135.644 \\
\hline 30 & 0.343 & 77.018 & 0.683 & 137.019 \\
\hline 60 & 0.683 & 77.047 & 0.509 & 138.602 \\
\hline
\end{tabular}


pressure, a novel approach for NO transport, local delivery, and triggered administration is of high clinical interest.

Paramount for successful diagnostics and therapy of myocardial ischemia is to achieve a biocompatible particle that can act both as a sensitive contrast agent for medical imaging as well as a drug carrier for efficient and controlled release of a therapeutic substance.

The current study expands the knowledge and further develops the multifunctional theranostic platform based on the polymer-shelled MB loaded with NO. Proposed multifunctional polymer MBs can be manufactured according to clinical requirements of UCAs with controlled size and narrow size distribution for improved ultrasound imaging. Moreover, polymer MBs are more stable, both chemically and mechanically, than currently clinically available contrast agents and can be loaded with NO for local and specific therapy. The thick shell of polymer MBs offers unique possibility for anchoring ligands for targeted visualization and therapy. However, the echogenicity of the polymer contrast is also reduced. This limitation was successfully overcome by the application of the multipulse technique in combination with chirp. ${ }^{44}$ As a result, nondestructive visualization of the thick-shelled MBs becomes possible. In addition, biotoxicity tests indicate that polymer MBs are biocompatible and phagocytosis begins within 6-8 hours. As a result, a single injection of multifunctional contrast agent together with ultrasound exposure has the potential to combine initial diagnostics with treatment and monitoring of the therapeutic effect.

\section{Disclosure}

The authors report no conflicts of interest in this work.

\section{References}

1. Allen TM, Cullis PR. Drug delivery systems: entering the mainstream. Science. 2004;303:1818-1822.

2. Mendis S, Puska P, Norrving B. Global Atlas on Cardiovascular Disease Prevention and Control. Geneva, Switzerland: World Health Organization; 2011.

3. Lindner JR. Microbubbles in medical imaging: current applications and future directions. Nat Rev Drug Discovery. 2004;3:527-533.

4. Cohen J, Cheirif J, Segar D, et al. Improved left ventricular endocardial border delineation and opacification with OPTISON (FS069), a new echocardiographic contrast agent: results of a phase III multicenter trial. J Am Coll Cardiol. 1998;32:746-752.

5. Schneider M. Characteristics of SonoVue. Echocardiography. 1999; 16:743-746.

6. Christiansen JP, Lindner JR. Molecular and cellular imaging with targeted contrast ultrasound. Proc IEEE. 2005;93:809-818.

7. Gorce JM, Arditi M, Schneider M. Influence of bubble size distribution on the echogenicity of ultrasound contrast agents: a study of Sonovue. Invest Radiol. 2000;35:661-671.

8. Klibanov AL. Microbubble contrast agent: targeted ultrasound imaging and ultrasound-assisted drug-delivery applications. Invest Radiol. 2006;41:354-362.
9. Leuschner F, Dutta P, Gorbatov R, et al. Therapeutic siRNA silencing in inflammatory monocytes in mice. Nat Biotechnol. 2011;29: 1005-1010.

10. Delalande A, Kotopoulis S, Rovers T, Pichon C, Postema M. Sonoporation at a low mechanical index. Bubble Sci Eng Technol. 2011; 3:3-12.

11. Darra E, Rungatscher A, Carcereri de Prati A, et al. Dual modulation of nitric oxide production in the heart during ischaemia/reperfusion injury and inflammation. Thromb Haemost. 2010;104:200-206.

12. Gonon AT, Jung C, Katz A, et al. Local arginase inhibition during early reperfusion mediates cardioprotection via increased nitric oxide production. PLoS One. 2012;7:e42038.

13. Palmer RM, Ferrige AG, Moncada S. Nitric oxide release accounts for the biological activity of endothelium-derived relaxing factor. Nature. 1986;327:524-526.

14. Radomski MW, Palmer RMJ, Moncada S. Endogenous nitric oxide inhibits human platelet adhesion to vascular endothelium. Lancet. 1987; 330:1057-1058.

15. Gaboury J, Woodman RC, Granger DN, Reinhardt P, Kubes P. Nitric oxide prevents leukocyte adherence: role of superoxide. Am J Physiol. 1993;265:H862-H862.

16. Luiking Y, Engelen M, Deutz N. Regulation of nitric oxide production in health and disease. Curr Opin Clin Nutr Metab Care. 2010;13:97-104.

17. Bertuglia S. Mechanisms by which low-intensity ultrasound improve tolerance to ischemia-reperfusion injury. Ultrasound Med Biol. 2007; 33:663-671.

18. Dörner J, Struck R, Zimmer S, et al. Ultrasound-mediated stimulation of microbubbles after acute myocardial infarction and reperfusion ameliorates left-ventricular remodelling in mice via improvement of borderzone vascularization. PloS One. 2013;8:e56841.

19. Postema M, Bouakaz A, ten Cate FJ, Schmitz G, De Jong N, van Wamel A. Nitric oxide delivery by ultrasonic cracking: some limitations. Ultrasonics. 2006;44:e109-e113.

20. Cavalieri F, El Hamassi A, Chiessi E, Paradossi G. Stable polymeric microballoons as multifunctional device for biomedical uses: synthesis and characterization. Langmuir. 2005;21:8758-8764.

21. Brismar TB, Grishenkov D, Gustafsson B, et al. Magnetite nanoparticles can be coupled with microbubbles to support multimodal imaging. Biomacromolecules. 2012;13:1390-1399.

22. Grishenkov D, Pecorari C, Brismar TB, Paradossi G. Characterization of acoustic properties of PVA-shelled ultrasound contrast agents: linear properties (Part I). Ultrasound Med Biol. 2009;35:1127-1138.

23. Pecorari C, Grishenkov D. Characterization of ultrasound-induced fracture of polymer-shelled ultrasonic contrast agents by correlation analysis. J Acoust Soc Am. 2007;122:2425-2430.

24. Govoni M, Jansson EA, Weitzberg E, Lundberg JO. The increase in plasma nitrite after a dietary nitrate load is markedly attenuated by an antibacterial mouthwash. Nitric Oxide. 2008;19:333-337.

25. Tortora G, Grabowski SR. Introduction to the Human Body. New York, USA: John Willey \& Sons, Inc.; 2001.

26. Baluk P, Hirata A, Thurston G, et al. Endothelial gaps: time course of formation and closure in inflamed venules of rats. Am J Physiol. 1997; 272:L155-L170.

27. Paradossi G, Pellegretti P, Trucco A. Ultrasound Contrast Agents. Targeting and Processing Methods for Theranostics. Milan, Italy: Springer-Verlag; 2010:25-39.

28. Zheng M. Ultrasound contrast agents: fabrication, size distribution and visualization. KTH DiVA. 2011.

29. De Jong N, Cornet R, Lancee CT. Higher harmonics of vibrating gas-filled microspheres. Part one: simulations. Ultrasonics. 1994;32: 447-453.

30. Cavalieri F, Finelli I, Tortora M, et al. Polymer microbubbles as diagnostic and therapeutic gas delivery device. Chem Mater. 2008;20: 3254-3258.

31. Poehlmann M, Grishenkov D, Kothapalli SVVN, et al. On the interplay of shell structure with low and high-frequency mechanics of multifunctional magnetic microbubbles. Soft Matter. 2014;10:214-226. 
32. Harrisson F, Callebaut M, Vakaet L. Microspectrographic analysis of Trypan Blue-induced fluorescence in oocytes of the Japanese quail. Histochemistry. 1981;72:563-578.

33. Paradossi G, Pellegretti P, Trucco A. Ultrasound contrast agents. Targeting and processing methods for theranostics. Milan, Italy: Springer-Verlag; 2010:67-77.

34. Barrefelt A, Saghafian M, Kuiper R, et al. Biodistribution, kinetics, and biological fate of SPION microbubbles in the rat. Int J Nanomedicine. 2013;8:3241-3254.

35. El-Sherif D, Wheatley MA. Development of a novel method for synthesis of a polymeric ultrasound contrast agent. J Biomed Mat Res A. 2003;66:347-355.

36. Grishenkov D, Gonon A, Paradossi G, Janerot-sjoberg B. Ultrasound contrast agent loaded with nitric oxide as a theranostic microdevice for myocardial ischemia. Proceedings of the Medicinteknikdagarna Conference; Oct 1-2, 2013, Stockholm, Sweden. Malmokongressbyra; 2013.

37. Morse PM, Ingard KU. Theoretical Acoustics. New York, USA: McGraw Hill; 1968.

38. Church $\mathrm{C}$. The effect of an elastic solid surface layer on the radial pulsations of gas bubbles. J Acoust Soc Am. 1995;97:1510-1521.
39. Kothapalli SVVN, Oddo L, Paradossi G, et al. Assessment of the viscoelastic and oscillation properties of a nanoengineered, shelled multimodality contrast agent. Ultrasound Med Biol. 2014;40:2476-2487.

40. Hoff L. Acoustic Characterization of Contrast Agents for Medical Ultrasound Imaging. Dordrecht, the Netherlands: Springer; 2001: 105-120.

41. Bamber JC, Hill CR. Ultrasonic attenuation and propagation speed in mammalian tissues as a function of temperature. Ultrasound Med Biol. 1979;5:149-157.

42. Marmottant P, van der Meer S, Emmer M, et al. A model for large amplitude oscillations of coated bubbles accounting for buckling and rupture. J Acoust Soc Am. 2005;118:3499-3505.

43. Wei K, Jayaweera AR, Firoozan S, et al. Quantification of myocardial blood flow with ultrasound-induced destruction of microbubbles administered as a constant venous infusion. Circulation. 1998;97:473-483.

44. Sciallero C, Paradossi G, Trucco A. A preliminary in vitro assessment of polymer-shelled microbubbles in contrast-enhanced ultrasound imaging. Ultrasonics. 2012;52:456-464.
Drug Design, Development and Therapy

\section{Publish your work in this journal}

Drug Design, Development and Therapy is an international, peerreviewed open-access journal that spans the spectrum of drug design and development through to clinical applications. Clinical outcomes, patient safety, and programs for the development and effective, safe, and sustained use of medicines are a feature of the journal, which

\section{Dovepress}

has also been accepted for indexing on PubMed Central. The manuscript management system is completely online and includes a very quick and fair peer-review system, which is all easy to use. Visit http://www.dovepress.com/testimonials.php to read real quotes from published authors.

Submit your manuscript here: http://www.dovepress.com/drug-design-development-and-therapy-journal 